Notwendig wäre eine echte stärkere Diskursivität in der politischen Kommunikation unter Wahlkampfbedingungen.

Deutlich zu sehen ist, dass die Zeit nach Ende des Wahlkampfs eine heikle Phase ist, wenn der politische Alltag wieder im Mittelpunkt steht. In dieser Phase, das gilt zumindest eindeutig für die Bundestagswahl von 2002, verschlechtern sich bei einem erheblichen Teil der Jugendlichen die zentralen politischen Einstellungen - offenbar aus Enttäuschung darüber, dass nur wenige der von den Parteien in Aussicht gestellten Modernisierungen umgesetzt werden können. Dann sind besondere kommunikative Kraftanstrengungen im Rahmen von Politikvermittlung und auch der politischen Bildungsarbeit sinnvoll, um diesen Effekt abzumildern und Verständnis für die komplexe Funktionslogik von Politik zu vermitteln.

\title{
Erfolgsbedingungen neuer Parteien im Parteiensystem am Beispiel der Piratenpartei Deutschland
}

\author{
Oskar Niedermayer
}

Sieht man vom Sonderfall der PDS/Linkspartei nach der deutschen Vereinigung ab, so hat es in der gesamten Geschichte des bundesrepublikanischen Parteiensystems nur eine neu gegründete Partei geschafft, sich auf Bundesebene dauerhaft als relevante Partei zu etablieren: die Grünen. Bedenkt man, dass in den letzten beiden Jahrzehnten neben den im Bundestag vertretenen Parteien im Schnitt 20 weitere an den Bundestagswahlen teilgenommen haben, so ist dies nicht gerade viel, und es stellt sich die Frage nach den Erfolgsbedingungen neuer Parteien im deutschen Parteiensystem. Da in neuerer Zeit von allen Kleinstparteien die Piratenpartei Deutschland bei weitem die größte Aufmerksamkeit erfahren hat und sie bei der Bundestagswahl 2009 unter den parlamentarisch nicht repräsentierten Parteien das mit Abstand beste Ergebnis verbuchen konnte, soll dieser Frage am Beispiel der Piratenpartei nachgegangen werden.

\section{Erfolg und Erfolgsbedingungen neuer Parteien im Parteiensystem}

Der „Erfolg“ einer neuen Partei wird hier durch das sukzessive Überschreiten von mehreren Karrierestufen gemessen, die qualitative Veränderungen der Rolle einer Partei im Parteiensystem markieren. Diese Entwicklung als „Lebenszyklus“1 zu sehen, ist in der Parteienforschung nicht neu. Eines der bekanntesten Modelle ist das „lifespan“-Modell von Pedersen²,

1 Im Lebenszyklus einer Partei geht es natürlich nicht nur aufwärts, es gibt auch Misserfolge, das heißt das Zurückfallen auf eine niedrigere Karrierestufe, bis hin zum „Tod“, das heißt zur Auflösung.

2 Vgl. Mogens N. Pedersen, Towards a New Typology of Party Lifespans and Minor Parties, in: Scandinavian Political Studies, 5. Jg. (1982), H. 1, S. 1 - 16, S. 6 f. 
der vier Karrierestufen unterscheidet: „declaration“ (die politische Gruppe entscheidet sich zur Wahlteilnahme), „authorization“ (die Partei wird zu einer Wahl zugelassen), „representation“ (die Partei ist parlamentarisch repräsentiert) und „relevance“ (sie verfügt über „coalition potential“ oder „blackmail potential“). Bei der Bestimmung der vierten Stufe knüpft er an Sartoris Kriterium für die Relevanz von Parteien an, nach dem eine parlamentarisch repräsentierte Partei dann als irrelevant angesehen werden kann, wenn sie kein Koalitionspotenzial hat, das heißt wenn ,it is never needed or put to use for any feasible coalition majority“. Unabhängig davon muss eine Partei jedoch immer dann als relevant angesehen werden, wenn sie Erpressungspotenzial hat, indem „,its existence, or appearance, affects the tactics of party competition"3. In Anlehnung an Pedersen und Lipset / Rokkan ${ }^{4}$ entwickelte Müller-Rommel für seine Analyse der Grünen in Westeuropa ein Modell, das von drei Hürden ausgeht, die überwunden werden müssen: die Legalitätshürde (Erfüllung der rechtlichen Voraussetzungen für die Nominierung von Kandidaten für lokale und regionale Wahlen), die Mobilisierungshürde (Aufbau einer nationalen Organisation und Nominierung von Kandidaten für nationale Parlamentswahlen) und die Repräsentationshürde (Vertretung im nationalen Parlament). ${ }^{5}$

$\mathrm{Zu}$ diesen Modellen lassen sich einige kritische Anmerkungen machen: Erstens umfassen politikwissenschaftliche Parteidefinitionen neben dem Kriterium der Organisiertheit in aller Regel auch das Kriterium der tatsächlichen Wahlteilnahme und nicht der bloßen Absicht, dies zu tun. ${ }^{6}$ Eine gesellschaftliche Organisation ist daher nicht schon deshalb eine Partei, weil sie sich so nennt beziehungsweise an Wahlen beteiligen möchte, sondern erhält diesen Status erst dann, wenn sie tatsächlich an einer Wahl teilnimmt. Zweitens kann eine Partei durchaus Erpressungspotenzial besitzen, ohne parlamentarisch repräsentiert zu sein. Drittens treten bei der Bestimmung der parlamentarischen Relevanz einer Partei nach ihrer Bedeutung für „feasible“ - also politisch machbare - Koalitionen des Öfteren Operationalisierungsprobleme auf. Als insofern problemlose Alternative bietet sich an, eine parlamentarisch vertretene Partei dann als relevant anzusehen, wenn mit ihr eine minimale Gewinnkoalition gebildet werden kann. Darunter wird im Rahmen der Koalitionstheorien ein Bündnis verstanden, das zum einen über eine Regierungsmehrheit verfügt (im Gegensatz zu einer Minderheitskoalition) und zum anderen eine minimale Größe in dem Sinne besitzt, dass jede Koalitionspartei zum Erreichen der Mehrheit benötigt wird (im Gegensatz zu einer übergroßen Koalition). Kann mit einer Partei eine minimale Gewinnkoalition gebildet werden, ist durch ihre Einbeziehung also rein rechnerisch die Bildung einer Mehrheitsregierung möglich, so ist diese Partei insofern relevant, als die für ein solches Bündnis in Frage kommenden anderen Parteien sie in ihre prinzipiellen Koalitionsüberlegungen einbeziehen und eine positive oder negative Koalitionsentscheidung treffen müssen. Ist dies nicht der Fall, dann ist die Partei für Regierungsbildungsprozesse vollkommen irrelevant.

3 Vgl. Giovanni Sartori, Parties and Party Systems. A Framework for Analysis, Cambridge 1976, S. $122 \mathrm{f}$.

4 Vgl. Seymour Martin Lipset / Stein Rokkan, Cleavage Structures, Party Systems, and Voter Alignments: An Introduction, in: dies. (Hrsg.), Party Systems and Voter Alignments. Cross-national Perspectives, New York 1967, S. 1 - 64.

5 Vgl. Ferdinand Müller-Rommel, Grüne Parteien in Westeuropa, Opladen 1993, S. 34.

6 Vgl. hierzu Oskar Niedermayer, Parteien, in: Dieter Fuchs / Edeltraud Roller (Hrsg.), Lexikon Politik, Stuttgart 2007, S. $195-197$. 
Viertens hat eine Partei mit dem Status als relevante Parlamentspartei noch nicht die höchste Stufe der Karriereleiter erreicht. Dies ist erst mit einer Regierungsbeteiligung der Fall. Wir unterscheiden daher in unserem Modell zur Messung des Erfolgs einer neuen Partei im Parteiensystem fünf Karrierestufen:

1. Wahlteilnahme (Zuerkennung der Parteieigenschaft durch die Zulassung zu einer Parlamentswahl - in der Bundesrepublik die Zulassung zu einer Landtags- oder Bundestagswahl);

2. Wettbewerbsbeeinflussung (die Existenz oder Aktivitäten der Partei führen zu Reaktionen anderer Parteien im Parteienwettbewerb). Die neue Partei erhält damit parteistrategische Relevanz für ihre Konkurrentinnen.

3. Parlamentarische Repräsentation (Einzug in ein Parlament, das heißt in Deutschland in einen Landtag, in den Bundestag oder in das Europäische Parlament). Dies ist der Indikator für elektorale Relevanz, das heißt für die Unterstützung durch einen relevanten Teil der Wählerschaft.

4. Koalitionsstrategische Inklusion (mit der Partei können rein rechnerisch minimale Gewinnkoalitionen gebildet werden). Damit erhält die Partei gouvernementale Relevanz, das heißt sie wird in Überlegungen zur Regierungsbildung einbezogen. ${ }^{7}$

5. Regierungsbeteiligung (die Partei wird zur Regierungspartei und hat damit die höchste Stufe ihrer Karriere erreicht).

Als Erfolgsbedingungen lassen sich diejenigen Faktoren ansehen, die das Überschreiten der verschiedenen Karrierestufen fördern oder behindern. Die Strukturierung der Fülle dieser Faktoren wird vereinfacht, wenn man sich vergegenwärtigt, dass für Parteiensysteme in demokratischen Gesellschaften der Wettbewerb zwischen den Parteien konstitutiv ist und jede Art von Wettbewerb durch Angebot, Nachfrage und Rahmenbedingungen bestimmt wird. Die Angebotsseite des politischen Wettbewerbs bilden die einzelnen Parteien, die durch den Einsatz von Ressourcen, durch inhaltliche Politikangebote und mit Hilfe unterschiedlicher Strategien versuchen, die Wahl in ihrem Sinne zu beeinflussen beziehungsweise für sich zu entscheiden. Die Nachfrageseite wird durch die Orientierungen und Verhaltensweisen der Wahlberechtigten gebildet. Der politische Wettbewerb findet zudem unter Rahmenbedingungen statt, die von den Wettbewerbsteilnehmern einerseits beachtet werden müssen, andererseits aber auch zum Teil - zumindest bis zur nächsten Wahl - beeinflusst werden können.

Im Einzelnen wirken die folgenden Faktoren auf der Angebotsseite des Parteienwettbewerbs auf den Erfolg einer neuen Partei:

(1) Die Ressourcen der Partei und ihrer Konkurrentinnen, insbesondere:

- Organisationsstrukturen (regionale und lokale Präsenz von Organisationseinheiten beziehungsweise die Existenz funktionaler Äquivalente für eine territoriale Organisation);

- Mitglieder (Anzahl der Parteimitglieder und sozialstrukturelle Zusammensetzung der Mitgliedschaft);

- Personal (Bekanntheit und Medientauglichkeit des Spitzenpersonals);

- Finanzausstattung (Höhe der selbsterwirtschafteten Einnahmen und Teilnahme an der öffentlichen Teilfinanzierung);

7 Natürlich können diese Überlegungen bei den potenziellen Koalitionspartnern auch zu der Schlussfolgerung führen, dass man mit dieser Partei keine Koalition eingehen kann. 
- Motivation, Willensbildungsprozesse und Strategiefähigkeit (Motivation zur Behauptung beziehungsweise Stärkung der Rolle der neuen Partei; Ausgestaltung der parteiinternen Willensbildungs- und Entscheidungsprozesse; Existenz eines strategischen Zentrums zur Steuerung der Partei im Wettbewerb).

(2) Die Strategien der Partei und ihrer Konkurrentinnen:

- Inhaltliche und organisatorische Strategien der Parteien zur Wählermobilisierung;

- Strategien der Konkurrenzparteien gegenüber der neuen Partei (inhaltliche Reaktionen: Ignorierung, Stigmatisierung, Thematisierung, Auseinandersetzung, Annäherung, Übernahme; organisationsbezogene Reaktionen: Kooperation, Eingliederung, Fusion).

(3) Die inhaltlichen Politikangebote der Partei und ihrer Konkurrentinnen:

- Verortung der Parteien auf den wesentlichen Konfliktlinien, die den Parteienwettbewerb prägen;

- Haltung zu einzelnen relevanten Themen.

Auf der Nachfrageseite des Parteienwettbewerbs können folgende Faktoren den Erfolg einer neuen Partei beeinflussen:

(1) Das Ausmaß und die Stärke von langfristigen Parteibindungen;

(2) die generellen Orientierungen gegenüber neuen Parteien;

(3) die Existenz, Veränderung und Herausbildung zentraler gesellschaftlicher Konfliktlinien, die auf unterschiedlichen Wertorientierungen beruhen;

(4) die Interessen und Organisierbarkeit bestimmter Gruppen in der Bevölkerung;

(5) die Orientierungen gegenüber einzelnen politischen Themen (Relevanz, inhaltliche Orientierung, Kompetenzzuweisung, Katalysatorthemen);

(6) die Orientierungen gegenüber dem politischen Spitzenpersonal der Parteien.

$\mathrm{Zu}$ den Rahmenbedingungen des Parteienwettbewerbs, die über den Erfolg einer neuen Partei mitbestimmen können, gehören:

(1) Der rechtliche Rahmen:

- Parteienrecht (insbesondere die Regelungen zur Gründung und zum Verbot von Parteien sowie die Ausgestaltung der öffentlichen Parteienfinanzierung);

- Wahlrecht (Regeln für die Wahlteilnahme und die parlamentarische Repräsentation);

- Staatsorganisation (Föderalismus oder Unitarismus);

(2) die Situation im übrigen intermediären System, insbesondere das Ausmaß der Unterstützung durch Medien, Verbände und soziale Bewegungen;

(3) ökonomische, soziale, demographische, innen- und außenpolitische, ökologische und technologische Entwicklungen beziehungsweise Ereignisse, die zu Veränderungen der inhaltlichen Positionen beziehungsweise Strategien der Parteien oder der Orientierungen beziehungsweise Verhaltensweisen der Bürger führen.

Die Relevanz der verschiedenen Erfolgsbedingungen variiert dabei stark je nach dem Typ der neuen Kleinpartei, wobei sich Ein-Thema-Parteien, Klientelparteien, Cleavage-Repräsentationsparteien, ideologische Nischenparteien, personenbezogene Parteien und Regionalparteien unterscheiden lassen. ${ }^{8}$

8 In der Parteienforschung gibt es keinen Konsens über die Typenbildung bei neuen Kleinparteien. Für eine Typologie, die sich an den Entstehungsursachen der neuen Partei orientiert, vgl. zum Beispiel Paul Lucardie, Prophets, Purifiers and Prolocutors. Towards a Typology on the Emergence of New Parties, in: Party Politics, 6. Jg. (2000), H. 2, S. $175-185$. 


\section{Die bisherige Karriere der Piratenpartei Deutschland}

Die Piratenpartei Deutschland (Piraten) wurde am 10. September 2006 in Berlin nach dem Vorbild der im Januar 2006 in Schweden entstandenen Piratpartiet gegründet. ${ }^{9}$ Sie versteht sich als Teil einer internationalen Bewegung zur Mitgestaltung des von ihr mit dem Terminus der „digitalen Revolution" 10 umschriebenen Wandels zur Informationsgesellschaft. Die erste Karrierestufe erreichten die Piraten mit der Zulassung ihrer Landesliste zur Teilnahme an der hessischen Landtagswahl im Januar 2008. Danach hat die Partei bis Mitte 2010 an fünf von zehn weiteren Landtagswahlen teilgenommen, wobei sie in Sachsen und Schleswig-Holstein 2009 mit etwas weniger als zwei Prozent die größten Erfolge verbuchen konnte (vgl. Tabelle 1).

Im Jahr 2009 nahm sie auf der Bundesebene sowohl an der Wahl zum Europäischen Parlament (EP) als auch an der Bundestagswahl teil. Mit der Zuerkennung der Parteieigenschaft durch den Bundeswahlausschuss und damit der Zulassung zur Bundestagswahl $2009^{11}$ haben die Piraten die erste Karrierestufe auch für die wichtigste Politikebene überschritten.

\begin{tabular}{|l|cc|}
\hline \multicolumn{3}{|c|}{ Tabelle 1: Wahlteilnahme und Wahlergebnisse der Piratenpartei seit ihrer Gründung } \\
am 10. September 2006 (Landtags-, Bundestags- und & EP-Wablen, in Prozent) \\
\hline & $\begin{array}{c}\text { Zweit- } \\
\text { stimmen }\end{array}$ & $\begin{array}{c}\text { Erst- } \\
\text { stimmen }\end{array}$ \\
\hline LTW Berlin 9/2006: Meldefrist verstrichen & - & - \\
LTW Mecklenburg-Vorpommern 9/2006: Meldefrist verstrichen & - & - \\
LTW Bremen 5/2007: keine Beteiligungsanzeige & - & - \\
LTW Hessen 1/2008 & 0,3 & - \\
LTW Niedersachsen 1/2008: zu wenig Unterstützungsunterschriften & - & - \\
LTW Hamburg 2/2008 & 0,2 & 0,1 \\
LTW Bayern 9/2008: zu wenig Unterstützungsunterschriften & - & - \\
LTW Hessen 1/2009 & 0,5 & - \\
EP-Wahl 6/2009 & & 0,9 \\
LTW Saarland 8/2009: keine Beteiligungsanzeige & - & - \\
LTW Sachsen 8/2009 & 1,9 & - \\
LTW Thüringen 8/2009: keine Beteiligungsanzeige & - & - \\
LTW Brandenburg 9/2009: keine Beteiligungsanzeige & - & - \\
LTW Schleswig-Holstein 9/2009 & 1,8 & 1,3 \\
BTW 9/2009 & 2,0 & 0,1 \\
LTW Nordrhein-Westfalen 5/2010 & 1,6 & 0,9 \\
\hline Quelle: Amtliche Wahlstatistiken. & & \\
\hline
\end{tabular}

9 Der Name Piratenpartei spielt auf die Kampagne von Musik- und Filmindustrieverbänden an, in der Verstöße gegen Urheberrechtsgesetze „Raubkopien“ (englisch: Piracy) genannt wurden. Zu den Anfängen der Partei vgl. Henning Bartels, Die Piratenpartei. Entstehung, Forderungen und Perspektiven der Bewegung, Berlin 2009.

10 Vgl. die Präambel des Grundsatzprogramms der Piratenpartei Deutschland.

11 Die Zulassung durch den Bundeswahlausschuss bedeutet nicht automatisch, dass eine Partei auch in allen Bundesländern an der Wahl teilnehmen kann, da bei parlamentarisch noch nicht repräsentierten Parteien die von ihnen eingereichten Landeslisten in den einzelnen Bundesländern von den Landeswahlausschüssen nur zugelassen werden, wenn die förmlichen Voraussetzungen erfüllt sind (insbesondere müssen die erforderlichen Unterstützungsunterschriften beigelegt sein). 
Die zweite Karrierestufe, die Wettbewerbsbeeinflussung durch die Reaktion der Konkurrenzparteien, überschritt die Partei spätestens nach der Bundestagswahl 2009. Dort hatte sie - mit dem Kampf gegen den von der CDU-Familienministerin Ursula von der Leyen initiierten Versuch, ein „Gesetz zur Bekämpfung von Kinderpornografie in Kommunikationsnetzen" auf den Weg zu bringen, als zentralem Wahlkampfthema - relativ gut abgeschnitten. Sie erhielt mit insgesamt 2,0 Prozent unter den parlamentarisch nicht repräsentierten Parteien den größten Wählerzuspruch, wobei sie vor allem bei den jüngeren Wählern punktete (vgl. Tabelle 2) und bei den 18- bis 24-jährigen Männern mit 12 Prozent gleichauf mit den Grünen zur viertstärksten Partei wurde.

\begin{tabular}{|c|c|c|c|}
\hline $\begin{aligned} \text { Tabelle 2: Wabla } & \\
& \text { (in Pro }\end{aligned}$ & & stagswahl 2009 nach Bevölkerungsg & \\
\hline Wahlergebnis insge & & & \\
\hline Geschlecht & & Geschlecht und Alter & \\
\hline Frauen & 1 & Frauen 18-24 Jahre & 5 \\
\hline Männer & 3 & Frauen 25-34 Jahre & 2 \\
\hline & & Frauen 35-44 Jahre & 1 \\
\hline Alter & & Frauen 45-59 Jahre & 1 \\
\hline 18-24 Jahre & 9 & Frauen 60 Jahre und älter & 0 \\
\hline 25-34 Jahre & 4 & Männer 18-24 Jahre & 12 \\
\hline 35-44 Jahre & 2 & Männer 25-34 Jahre & 6 \\
\hline 45-59 Jahre & 1 & Männer 35-44 Jahre & 3 \\
\hline 60 Jahre und älter & 0 & Männer 45-59 Jahre & 1 \\
\hline & & Männer 60 Jahre und älter & 0 \\
\hline Berufstätigkeit & & & \\
\hline Arbeiter & 3 & Bildung & \\
\hline Angestellte & 3 & Abitur / Universität & 3 \\
\hline Beamte & 1 & Mittel- / Realschule & 2 \\
\hline Selbständige & 3 & kein Abschluss / Volks- / Hauptschule & 1 \\
\hline Arbeitslose & 3 & & \\
\hline
\end{tabular}

Diese Ergebnisse führten zu Reaktionen der anderen Parteien. Hatte man die Piraten vorher mehr oder weniger ignoriert, so betonte die Bundeskanzlerin Angela Merkel schon auf der Pressekonferenz am Tag nach der Bundestagswahl, dass man den Dialog mit ihren Wählern aufnehmen müsse. ${ }^{12}$ Die anderen Parteien übernahmen die Strategie der Thematisierung der Netzpolitik und gingen partiell auf bestimmte Forderungen der Piraten ein: Die Unionsfraktion schlug die Einrichtung einer Enquete-Kommission des Bundestags zum Thema „Internet und digitale Gesellschaft“ vor, die die Folgen der Online-Revolution für Gesellschaft, Wirtschaft und Recht erörtern und für den Bundestag Empfehlungen erarbeiten soll. Die anderen Fraktionen stimmten dem Vorschlag zu, und die Kommission nahm Anfang Mai 2010 ihre Arbeit auf. Die FDP-Fraktion gründete eine Querschnitts-AG

12 Vgl. Özlem Topcu, Bloß nicht offline, in: Die Zeit vom 1. Oktober 2009. 
„IT und Informationsgesellschaft“, die Regierung setzte das Netzsperren-Gesetz für ein Jahr aus, der CDU-Bundesinnenminister Thomas de Maizière begann, sich regelmäßig mit Netzaktivisten zu Diskussionsveranstaltungen zu treffen, die neue FDP-Bundesjustizministerin Sabine Leutheusser-Schnarrenberger setzte in der Netzpolitik neue Akzente ${ }^{13}$, und die SPD richtete einen Gesprächskreis „Netzpolitik und digitale Gesellschaft“ im Rahmen der Medienkommission des Parteivorstands ein, der den früheren Online-Beirat fortführt und momentan die netzpolitischen Leitlinien der SPD „erneuert“, „zum Teil auch revidiert“. ${ }^{14}$ Die Grünen bekannten sich unter dem Motto „Digital ist besser - Für ein freies Internet“ zur digitalen Revolution ${ }^{15}$ und gingen sogar in einem Fall über eine rein inhaltliche Reaktion hinaus, indem ihr thüringischer Landesverband vor der Landtagswahl 2009 in einem Kooperationspapier eine partielle Zusammenarbeit mit den nicht an der Wahl teilnehmenden Piraten in den Punkten Bürgerrechte und Freiheit vereinbarte.

Insgesamt blieb jedoch der Einfluss der Piraten auf den zwischenparteilichen Wettbewerb sehr begrenzt, wie auch der Wahlkampf zur ersten Landtagswahl nach der Bundestagswahl in Nordrhein-Westfalen zeigte. Insbesondere gab es keinerlei organisatorische Reaktionen, also Anstrengungen, die neue Partei einzugliedern beziehungsweise mit ihr zu fusionieren. Solche Reaktionen sind auch nur wahrscheinlich, wenn die neue Partei als relevante Konkurrentin um die eigene Wählerbasis perzipiert wird ${ }^{16}$, was bis jetzt nicht der Fall ist. Die Wählerwanderungsanalyse für die Bundestagswahl gibt dieser Ansicht recht: Die Piraten rekrutierten ihre Wähler nicht einseitig zu Lasten einer Partei, auch nicht der Grünen, wie oft vermutet wurde. In der Größenordnung von je einem Fünftel waren es Erstwähler und frühere SPD-Wähler, je etwa ein knappes Siebtel kamen von der Union und den Grünen, ungefähr je ein Zwanzigstel kamen von der FDP, der Linkspartei und sonstigen Parteien. ${ }^{17}$ Damit hält sich die bisherige Gefährdung der Wählerbasis der anderen Parteien durch die Piraten in sehr engen Grenzen. Insofern sind deren Reaktionen eher als Präventivmaßnahmen zu verstehen, die den Piraten den Wind aus den Segeln nehmen sollen, bevor sie eine relevante Größe im Parteienwettbewerb werden.

13 So will sie sich mit einer neuen gesetzlichen Regelung zum Thema Netzsperren „Zeit lassen“ und bei der Vorratsdatenspeicherung „nichts überstürzen“ und über ihrem Leitantrag auf dem jüngsten FDP-Bundesparteitag sah man „die schwarze Flagge der Piratenpartei geradezu im Wind knattern", Ulrich Clauß, Regeln für das Internet, in: Welt online vom 6. Mai 2010, http://www. welt.de/die-welt/debatte/article7496423/Regeln-fuer-das-Internet.html (Abruf am 25. Oktober 2010).

14 So der netzpolitische Sprecher des SPD-Parteivorstands, Björn Böhning, zitiert nach SPD-Netzpolitik, Schweres Erbe und neuer Kurs, http://www.politik-digital.de/parteien-im-netz-spd-netzpolitik (Abruf am 21. Juli 2010).

15 Vgl. Klare digitale Leitlinien bei den Grünen, http://www.politik-digital.de/parteien-im-netz-diegruenen-netzpolitik (Abruf am 13. Juli 2010).

16 Wie es zum Beispiel bei der 2004 gegründeten WASG der Fall war, die für die damalige PDS zur westdeutschen Konkurrentin zu werden drohte, aber auch die Chance bot, durch ein Zusammengehen das Vehikel für die erstrebte Westausdehnung zu bilden. Die PDS-Führung entschied sich sehr früh, nicht auf Konfrontationskurs zur WASG zu gehen, sondern eine Kooperation mit dem Ziel der Fusion anzustreben, die im Juni 2007 mit der Gründung der neuen Partei Die Linke formell abgeschlossen wurde.

17 Ergebnisse der Wählerwanderungsanalyse von Infratest dimap, die dem Verfasser freundlicherweise zur Verfügung gestellt wurden. 


\section{Erklärung des bisherigen Erfolgs und Zukunftsaussichten}

Um den bisherigen, durch das Überschreiten der zweiten Karrierestufe dokumentierten Erfolg der Piraten zu erklären und ihre Zukunftsaussichten zu beurteilen, müssen Erfolgsfaktoren aus allen drei im Abschnitt 2 vorgestellten Bereichen herangezogen werden.

\subsection{Die Ressourcen und Strategien der Piraten und ihrer Konkurrentinnen}

Auf der Angebotsseite des Parteienwettbewerbs ist zunächst zu konstatieren, dass die Piraten nach ihrer Gründung Ende 2006 relativ schnell die erforderlichen parteiorganisatorischen Voraussetzungen für das Überschreiten der ersten Karrierestufe geschaffen haben. Obwohl sie in ihren gesamten Aktivitäten sehr stark netzfixiert sind, haben sie dem nach dem Parteien- und Wahlgesetz für eine Wahlteilnahme notwendigen Territorialprinzip durch den schnellen Aufbau einer territorial gegliederten Parteiorganisation Rechnung getragen: Schon Ende 2007 waren sie in der Hälfte der Bundesländer mit eigenen Landesverbänden vertreten, und seit dem Achtungserfolg bei den EP-Wahlen im Juni 2009 verfügen sie auf der Landesebene über eine flächendeckende territoriale Organisationsstruktur, was sie von den meisten anderen Kleinstparteien unterscheidet. ${ }^{18}$ Diesen sind sie auch in der Mitgliederstärke in neuerer Zeit um Längen voraus. ${ }^{19}$ Nach einer längeren Phase mäßiger Aufwärtsentwicklung brachten die EP- und Bundestagswahl einen enormen Mitgliederschub. Allerdings stagniert die Mitgliederzahl seit dem Jahresbeginn 2010 bei etwa 12.000 (vgl. Abbildung 1).

Weniger positiv sieht es beim Spitzenpersonal aus, wo sich Ressourcenstärke an der Bekanntheit und Medientauglichkeit misst. Zwar finden sich mit Angelika Beer und Herbert Rusche eine frühere kurzzeitige Grünen-Vorsitzende und ein früherer Grünen-MdB in ihren Reihen, beide sind der Öffentlichkeit jedoch heutzutage fast völlig unbekannt, was auch für den gesamten Vorstand der Partei gilt. Den Piraten fehlt also ein Gesicht, das für sie steht. Ihr mit Abstand bekanntestes Mitglied war der langjährige Sprecher der AG Bildung und Forschung der SPD-Bundestagsfraktion, Jörg Tauss, der im Juni 2009 aus Protest gegen das geplante Gesetz zur Bekämpfung von Kinderpornografie in Kommunikationsnetzen seine Partei verließ und sich demonstrativ der Piratenpartei anschloss, so dass ein Mitglied der Piraten als fraktionsloser Abgeordneter bis zur Wahl im September 2009 im Bundestag saß. Allerdings ermittelte parallel hierzu die Staatsanwaltschaft gegen ihn wegen des Verdachts auf Besitz kinderpornografischer Schriften. Als Tauss Ende Mai 2010 zu einer - mittlerwei-

18 Auch die weitere satzungsmäßige Untergliederung der Parteiorganisation in - bisher noch in geringem Maße vorhandene - Bezirks-, Kreis- und Ortsverbände richtet sich an der Territorialgliederung der Bundesrepublik aus, zusätzlich sind in Berlin und NRW „Crews“ genannte Basisgruppen ein förmlicher Teil der Parteigliederung.

19 Dies gilt für die Kleinstparteien, das heißt Parteien, die noch nie in einem Landtag, dem Bundestag oder dem Europäischen Parlament vertreten waren, und darüber hinaus auch für die auf Landesebene temporär und partiell repräsentierten Kleinparteien wie die rechtsextremen Parteien NPD, DVU und Republikaner, nicht jedoch für die kleineren im Bundestag repräsentierten Parteien, die deutlich über den Mitgliederzahlen der Piraten liegen. Die Linke hatte Ende 2009 78.496, die FDP 72.116 und die Grünen 48.171 Mitglieder, vgl. Oskar Niedermayer, Die Entwicklung der Parteimitgliedschaften von 1990 bis 2009, in: ZParl, 41. Jg. (2010), H. 2, S. 421 -437, S. 425. 


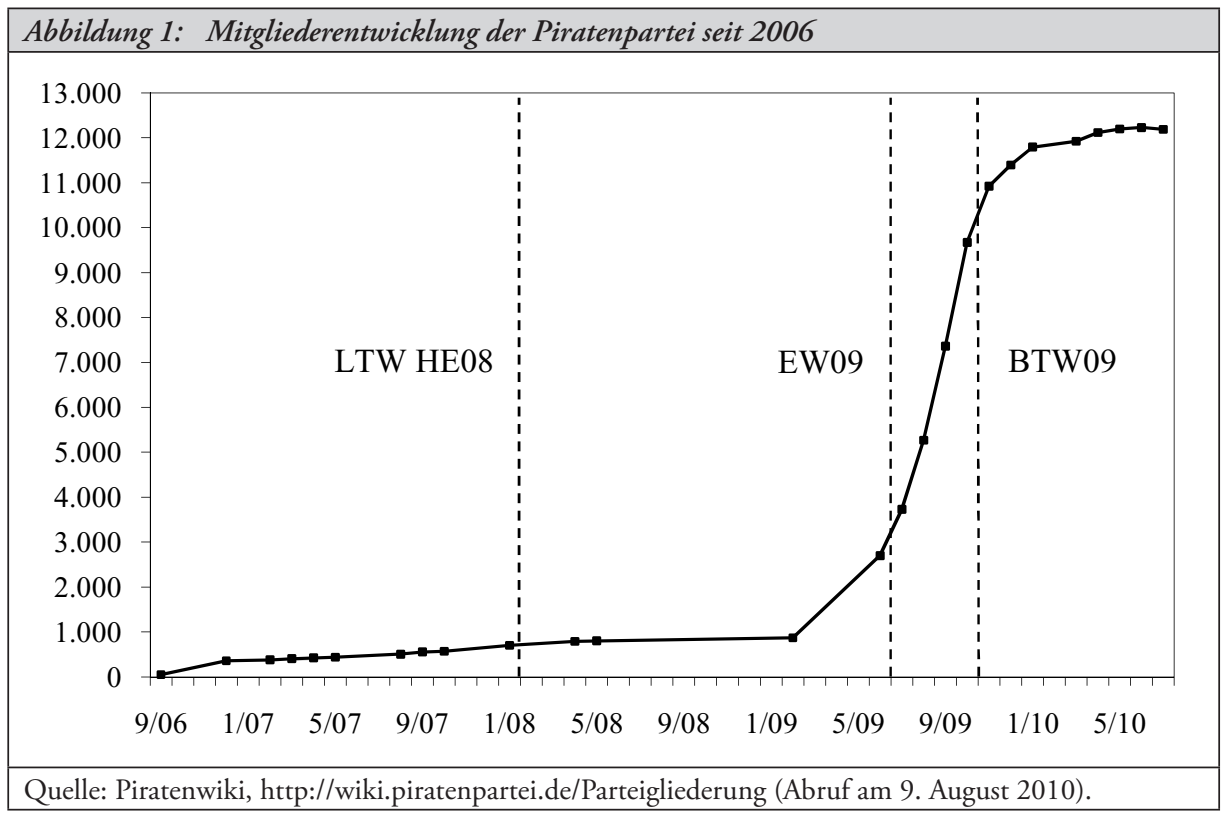

le rechtskräftigen - Bewährungsstrafe von 15 Monaten verurteilt wurde, trat er aus der bis zuletzt hinter ihm stehenden Partei aus. ${ }^{20}$

Auch die Finanzausstattung der Piraten lässt zu wünschen übrig. Die selbst erwirtschafteten Einnahmen der Partei in Form von Mitgliedsbeiträgen und Spenden halten sich in engen Grenzen, und die Partei erhielt erst für das Jahr 2009 öffentliche Mittel, da nur diejenigen Parteien Anspruch auf staatliche Teilfinanzierung in einem bestimmten Jahr haben, die bei der letzten Bundestags- oder EP-Wahl mindestens 0,5 Prozent beziehungsweise bei einer der letzten 16 Landtagswahlen mindestens 1 Prozent der abgegebenen gültigen Listenstimmen auf sich vereinigen konnten, wobei sich die Höhe der Mittel nach dem Wahlerfolg und den Eigeneinnahmen der jeweiligen Partei richtet. Nach der Anzahl der Wählerstimmen, die die Piratenpartei bis Ende 2009 bei der Bundestags- und EP-Wahl 2009 sowie den Landtagswahlen 2009 in Sachsen und Schleswig-Holstein erhielt (1.140.822 Stimmen) und der Höhe der berücksichtigungsfähigen Zuwendungen des Jahres 2008 (21.946,27 Euro) hätte die Partei Anspruch auf staatliche Mittel in Höhe von 840.554,51 Euro gehabt. Wegen des aus dem Grundgesetz abgeleiteten Verbots einer überwiegenden staatlichen Parteienfinanzierung darf diese jedoch nicht höher sein als die von den Parteien selbst erwirtschafteten Eigeneinnahmen des Vorjahres. Da die Piratenpartei in ihrem Rechenschaftsbericht für 2008 nur 31.504,68 Euro Eigeneinnahmen ausgewiesen hatte, wurden die staatlichen Mittel auf diese Höhe begrenzt. ${ }^{21}$ So plagten auch die Partei in Nordrhein-Westfalen im

20 Vgl. Ole Reißmann, Tauss verlässt die Piratenpartei, in: Spiegel online vom 30. Mai 2010, http:// www.spiegel.de/politik/deutschland/0,1518,697599,00.html (Abruf am 25. Oktober 2010).

21 Zum Vergleich: die FDP erhielt 12,6 Millionen, die Grünen 11,1 Millonen und die Linkspartei 10,7 Millonen Euro. Von den bei den letzten Wahlen deutlich weniger erfolgreichen Kleinstparteien erhielten zum Beispiel die Republikaner 1,2 Millionen und die ödp 700.000 Euro. 
Landtagswahlkampf „notorische Geldnöte“22, die die Organisation eines flächendeckenden Wahlkampfes schwer machten. Der Kontostand des Bundesverbands betrug Ende Juli 2010 gerade einmal knapp 81.000 Euro, wobei von diesem Geld vieles schon verplant war. ${ }^{23}$

Bei der Bewertung der Rolle der finanziellen Ressourcen für die bisherigen und zukünftigen (Wahl-)Erfolge der Partei ist einerseits zu bedenken, dass die primäre Ausrichtung auf den basiszentrierten Online-Wahlkampf die Bedeutung der Finanzausstattung relativiert, andererseits erschwert ein weitgehender Verzicht auf „paid-media“ und weitere kostenträchtige Wahlkampfmittel erheblich die Mobilisierung von Randwählern.

Insgesamt lässt sich konstatieren, dass die Piraten über ausreichende organisatorische Ressourcen verfügen, um die Parteiorganisation zu verstetigen und kampagnenfähig zu halten. Zudem haben sie einen deutlichen Kompetenzvorsprung in der zielgruppenadäquaten inhaltlichen und organisatorischen Strategie der Wählermobilisierung: Die Partei machte im Bundestagswahlkampf den anderen Parteien vor, „wie man im Internet Wähler für sich begeistern kann“ 24 , indem sie „als einzige politische Organisation fast alle zur Verfügung stehenden interaktiven Optionen“ 25 in ihrer Kampagne einsetzte und „vielleicht den einzig ,echten’ Mitmach-Wahlkampf im Web 2.0“26 führte. Allerdings wies sie im Offline-Wahlkampf noch deutliche Schwächen auf: Für den Flächenwahlkampf fehlte es an Geld, für den Straßenwahlkampf an Erfahrung, auch wenn man mit einigen ungewöhnlichen Flashmob-Aktionen auch offline auf sich aufmerksam machte. Dass lokale Präsenz und offlineAktivitäten durchaus Wählerstimmen bringen, zeigt die NRW-Landtagswahl. In Aachen, wo die Piraten mit einem Stadtrat lokalpolitisch vertreten sind, holten sie mit 4,2 Prozent (Wahlkreis Aachen I) und 3,1 Prozent (Aachen II) ihr landesweit bestes Ergebnis, und in Köln, wo man auf der Straße aktiv war, kam man auf weit überdurchschnittliche Ergebnisse von bis 2,3 Prozent. ${ }^{27}$

Die Strategien der Konkurrenz gegenüber der Piratenpartei wurden im Abschnitt 2 schon verdeutlicht, und an Motivation zur weiteren Teilnahme am Parteienwettbewerb fehlt es den Piraten nicht, wie ihr Vorhaben, an allen Landtagswahlen des Jahres 2011 teilzunehmen, zeigt. Als letzter Ressourcenfaktor ist somit die Ausgestaltung des innerparteilichen Willensbildungs- und Entscheidungsprozesses und die damit eng zusammenhängende Frage nach der Existenz eines strategischen Zentrums zur Steuerung der Partei im Wettbewerb zu betrachten.

Das originäre Kommunikations-, Partizipations- und Organisationsverständnis der Piratenpartei beruht auf den Prinzipien der Transparenz, Inklusion und Entgrenzung, das heißt

22 Martin Teigeler, Jung und Klamm: Piratenpartei NRW plagen notorisch Geldnöte, in: Aachener Nachrichten vom 23. Dezember 2009.

23 Vgl. Piratenpartei, Kontostand Bundesverband, http://wiki.piratenpartei.de/Kontostand (Abruf am 9. August 2010).

24 Konrad Fischer / Oliver Voß, Die Piratenpartei dominiert den Wahlkampf im Netz, in: Wirtschaftswoche vom 23. August 2009.

25 Eva Johanna Schweitzer, Normalisierung 2.0. Die Online-Wahlkämpfe deutscher Parteien zu den Bundestagswahlen 2002-2009, in: Christina Holtz-Bacha (Hrsg.), Die Massenmedien im Wahlkampf. Das Wahljahr 2009, Wiesbaden 2010, S. 189 - 244, S. 217.

26 Christoph Bieber, Kampagne als „Augmented Reality Game“: Der Mitmachwahlkampf der Piratenpartei, http://carta.info/15450/kampagne-als-augmented-reality-game-der-mitmachwahlkampf-der-piratenpartei (Abruf am 25. September 2009).

27 Auch in Münster, wo die Partei ihren zweiten Stadtrat besitzt, holte sie ein deutlich überdurchschnittliches Ergebnis, vgl. Die Landeswahlleiterin des Landes Nordrhein-Westfalen, Landtagswahl 2010, Heft 3: Endgültige Ergebnisse in Nordrhein-Westfalen, Düsseldorf 2010. 
die Willensbildungs- und Entscheidungsprozesse sollen möglichst offen, mit möglichst breiter Beteiligung und nicht auf die Parteimitglieder beschränkt ablaufen. Für einige Beobachter ist die Piratenpartei damit die erste Parteiorganisation, die „organisatorisch Konsequenzen aus dem Niedergang etablierter Organisationen und dem Erfolg unkonventioneller Bündnisse“ zieht, was ihre „besondere Stärke“28 ausmacht und damit wesentlich zu ihrem Erfolg beiträgt. Nun mag es ja durchaus sein, dass diese Organisationsprinzipien für viele Menschen den wesentlichen Anreiz lieferten, sich den Piraten zuzuwenden beziehungsweise bei ihnen mitzumachen; für reine Willensbildungsprozesse mögen sie adäquat sein, obwohl sich einem Beobachter der zuweilen chaotisch ablaufenden und seltsame Blüten treibenden ${ }^{29}$ Diskussionen in den verschiedenen Netzforen der Piraten in dieser Hinsicht ernste Zweifel aufdrängen. Für Entscheidungsprozesse sind dem Erfolg einer solchen Organisationsform jedoch durch die rechtlichen Bestimmungen zur innerparteilichen Organisation und durch die Erfordernisse des Parteienwettbewerbs momentan noch enge Grenzen gesetzt. So müssen inhaltliche und personelle Entscheidungen laut Parteien- und Wahlgesetz durch eindeutig definierte Gremien und nach Verfahren mit einem eindeutigen Raum-Zeit-Bezug getroffen werden. Das höchste Gremium für inhaltliche Grundentscheidungen ist der Bundesparteitag, der zwar nicht unbedingt - dem Repräsentationsprinzip folgend - aus gewählten Delegierten bestehen muss, sondern auch - wie bei den Piraten eine Mitgliederversammlung auf Bundesebene sein kann, aber die Entscheidungen werden eben von Mitgliedern - nicht von Außenstehenden - getroffen, und zwar nur von denjenigen, die dem zu einem bestimmten Zeitpunkt tagenden Parteitag angehören.

Die Piratenpartei hat diesem Erfordernis bei der Neuorganisation ihrer inhaltlichen Entscheidungsprozesse Rechnung getragen und nutzt dabei die Möglichkeiten der neuesten Kommunikationstechnologie. Auf dem Bundesparteitag am 15./16. Mai 2010 in Bingen am Rhein wurde die Einführung des elektronischen Diskussions- und Abstimmungssystems LiquidFeedback beschlossen, das „als konstruktiver Diskussionsraum einen Kontrast zu den teilweise destruktiven Auseinandersetzungen auf den Mailinglisten der Partei bilden "30 soll. Nach einer auch nach dem Parteitag weitergeführten kontroversen Diskussion um Nutzungsbedingungen und Datenschutz, die tiefe Gräben in der Partei aufriss und zum Rücktritt eines Bundesvorstandsmitglieds führte, wurde das System Mitte August 2010 eingeführt. ${ }^{31}$ Mit LiquidFeedback sollen bundesweit alle Parteimitglieder - und nur diese - dauerhaft in die Entscheidungen der Partei eingebunden werden. ${ }^{32}$ Das System ermöglicht in einer flexiblen Mischform aus direkter und repräsentativer Demokratie, inhaltliche Vorschläge einzubringen, bei den anderen Mitgliedern um Zustimmung zu werben, sie breit zu diskutieren, laufend zu verändern und sie letztlich abzustimmen, wobei jedes

28 Felix Neumann, Piratenpartei: Mehr als die Autofahrerpartei auf der Datenautobahn, http://carta. info/22886/piratenpartei-autofahrerpartei-auf-der-datenautobahn (Abruf am 12. Februar 2010).

29 So zum Beispiel im Umgang mit Familienministerin Ursula von der Leyen („Zensursula“) während der Diskussion um das Netzsperrengesetz.

30 Torsten Kleinz, Piratenpartei auf der Gratwanderung zwischen Transparenz und Datenschutz, http://www.heise.de/newsticker/meldung/Piratenpartei-auf-der-Gratwanderung-zwischen-Transparenz-und-Datenschutz-1051745.html (Abruf am 6. August 2010).

31 Vgl. Piraten starten Großversuch zu direkter Demokratie, Pressemitteilung der Piratenpartei Deutschland vom 13. August 2010.

32 Da das System teilweise auch von außen einsehbar ist, können Nichtmitglieder verfolgen, worüber die Partei debattiert und wie sie abstimmt, nicht jedoch selbst Entscheidungen treffen. 
Mitglied seine Stimme umfassend oder bei spezifischen Themen auch an andere delegieren kann. „Die in LiquidFeedback beschlossenen Anträge sind nicht bindend, sondern bilden lediglich das Meinungsbild der teilnehmenden Piraten ab. "33 Als Ergebnis des innerparteilichen Willensbildungs- und Entscheidungsprozesses sollen Meinungsbilder auf breiter Grundlage entstehen, die „für die Entscheidungen der Parteitage und Vorstände eine qualifizierte Grundlage bilden“34.

Mit der Einführung dieses Verfahrens sind jedoch bei Weitem nicht alle Probleme beseitigt. Es bestehen weiterhin drei gravierende Defizite, die die zukünftigen Erfolgschancen der Partei behindern: Erstens gibt es kein Verfahren der innerparteilichen Konfliktregulierung für den Fall, dass die Mehrheiten in den Parteigremien und im LiquidFeedback-Netzwerk nicht übereinstimmen. Zweitens fehlt der Partei angesichts eines Bundesvorstands, der die Partei nur verwaltet und allenfalls als unverbindliches Moderations- statt als politisches Führungsorgan agiert, ein „strategisches Entscheidungs- und Machtzentrum“35, um im Parteienwettbewerb in effektiver Weise strategisch handeln zu können. ${ }^{36}$ Drittens ist in einigen Bereichen, vor allem beim Umgang mit den finanziellen Ressourcen, eine Professionalisierung dringend geboten. ${ }^{37}$

\subsection{Die politischen Inhalte: Angebot und Nachfrage im Parteienwettbewerb}

Neben den Ressourcen und Strategien wird der Erfolg der Piraten im Parteienwettbewerb wesentlich von den inhaltlichen Politikangeboten der Partei und ihrer Konkurrentinnen und der Nachfrage nach diesen Angeboten auf Seiten der Wählerschaft bestimmt. Hier stellen sich zwei Fragen: (1) Kann eine neue Partei heutzutage angesichts der Orientierungen der Wahlberechtigten überhaupt zu einer relevanten Größe im Parteienwettbewerb werden? (2) Wie sieht das inhaltliche Politikangebot der Piraten konkret aus, und in welchem Ausmaß kann sie damit in Konkurrenz zu den anderen Parteien Wähler für sich gewinnen?

33 So der Antragstext in Piratenpartei Deutschland, Bundesparteitag 2010, Sonstiger Antrag Nr. Z013: Bundesweiter Betrieb von LiquidFeedback, http://wiki.piratenpartei.de/Bundesparteitag_2010.1/Protokoll (Abruf am 25. Oktober 2010).

34 Pressemitteilung der Piratenpartei, a.a.O. (Fn. 31).

35 Udo Zolleis / Simon Prokopf / Fabian Strauch, Die Piratenpartei. Hype oder Herausforderung für die deutsche Parteienlandschaft?, Hanns-Seidel-Stiftung, Aktuelle Analysen, Nr. 55, München 2009, S. 22.

36 „Diese Partei ist wie das Internet selbst: chaotisch und ungesteuert.“ Tillmann Prüfer, Klar zum Kentern, in: ZEITmagazin vom 6. Mai 2010.

37 So fand der neue Schatzmeister der Partei bei seinem Amtsantritt im Juli 2009 nach eigenen Angaben „statt gut geführter Bücher nur eine Kiste mit Zetteln“ vor und es fehlten seiner Schätzung nach „für 30 Prozent der Gesamteinnahmen Belege und Angaben über die Spender“ (zitiert nach Sonja Bechtold, Schatzmeister Schömers Wettlauf gegen die Zeit, in: Spiegel online vom 30. Dezember 2009). Auch Mitte 2010 war die Erfassung der Zahlungseingänge von Mitgliedern nach Berichten eines Insiders immer noch in einem "desolaten Zustand“ (vgl. Torsten Kleinz, a.a.O., Fn. 30). Ein solch leichtfertiger Umgang mit den Finanzen schädigt nicht nur die finanzielle Ressourcenbasis der Partei, da nicht belegte Einnahmen zum Beispiel nicht in den Rechenschaftsbericht aufgenommen und damit auch nicht bei der Zuteilung öffentlicher Gelder berücksichtigt werden können, sondern behindert auch ihre internen Entscheidungsprozesse, weil keine Klarheit über die Gesamtheit der Stimmberechtigten besteht. 
Die erste Frage ist mit „ja“ zu beantworten. Seit geraumer Zeit führen Veränderungen der Rahmenbedingungen des politischen Wettbewerbs durch langfristige Prozesse des ökonomischen, sozialen und kulturellen Wandels zu einer Aufweichung der Beziehung zwischen sozialer Gruppenzugehörigkeit und Wertorientierungen, zu einer Erosion der traditionellen sozialen Milieus und damit einer Schrumpfung der Kernwählergruppen der etablierten Parteien sowie generell zu einer Abnahme der langfristigen Parteibindungen. Insgesamt wurde damit das Wahlverhalten flexibilisiert, und dies bedeutet nicht nur, dass die etablierten Parteien immer weniger auf einen festen Wählerstamm setzen können und ihre Wähler bei jeder Wahl aufs Neue durch ein optimales personelles und inhaltliches Angebot überzeugen müssen, sondern auch, dass neue Parteien mit einem attraktiven Angebot im Parteienwettbewerb durchaus Chancen haben.

Um die zweite Frage zu beantworten, muss abgeschätzt werden, um welchen Typ von neuer Partei es sich bei den Piraten handelt. ${ }^{38}$ Sind sie eine reine Ein-Thema-Partei, deren Erfolg sich allein daran misst, in welchem Maße ihnen die Wähler dabei Kompetenz und Glaubwürdigkeit zuschreiben und wie relevant das Thema jeweils für die Entscheidung bei den verschiedenen Wahlen ist? Oder sind sie als Klientelpartei anzusehen, die die Interessen einer relativ klar umgrenzten Bevölkerungsgruppe im Rahmen der bestehenden gesellschaftlichen Konfliktstruktur vertritt und deren Erfolg damit von der Größe und Mobilisierbarkeit dieser Gruppe sowie von der gesellschaftlichen und parteipolitischen Relevanz des übergeordneten Konflikts abhängt? Oder stützen sie sich gar auf eine neu entstandene, zentrale gesellschaftliche Konfliktlinie, deren einen Pol sie parteipolitisch organisieren und damit als Cleavage-Repräsentationspartei in die parteipolitische Konfliktstruktur einbringen?

Unstrittig ist, dass die Piraten als Single-Issue-Party gestartet sind. Aus ihrem inhaltlichen Kernbereich, der Netzpolitik, stammt das „Katalysatorthema“, das ihnen Aufmerksamkeit sowohl innerhalb als auch außerhalb der Netz-Community eingebracht und ihren bisherigen Erfolg erst möglich gemacht hat: die Anfang 2009 von der CDU-Familienministerin Ursula von der Leyen zur Diskussion gestellte Erschwerung des Zugangs zu kinderpornografischen Inhalten im Internet, die am 18. Juni mit den Stimmen von Union und SPD als Zugangserschwerungsgesetz in Gesetzesform gegossen wurde. Die Piraten wandten sich nicht gegen den Zweck des Gesetzes, sahen aber den Weg zur Zielerreichung als unbrauchbar an, werteten es als Einstieg in eine Internetzensur und begannen den „Kampf gegen den herannahenden Überwachungsstaat“39. Mit ihrem Fokus auf die Freiheit im Netz trafen sie den Nerv vieler Jugendlicher, die sich „von der Politik gegängelt, überwacht und zu Unrecht kriminalisiert“ ${ }^{40}$ fühlten. Die kontroverse Diskussion um das Netzsperrengesetz thematisierte zum ersten Mal in einer breiteren Öffentlichkeit den

38 Hierzu bestehen in der Literatur unterschiedliche Auffassungen, vgl. zum Beispiel Fabian Blumberg, Partei der „digital natives“? Eine Analyse der Genese und Etablierungschancen der Piratenpartei, Konrad-Adenauer-Stiftung, Berlin 2010, S. 18 ff.; Marcel Solar, Klarmachen zum Ändern? Aufstieg und Perspektiven der deutschen Piratenpartei, in: Mitteilungen des Instituts für Parteienrecht und Parteienforschung (MIP), 16. Jg. (2010), S. 108 - 111, S. 110; Udo Zolleis I Simon Prokopf/ Fabian Strauch, a.a.O. (Fn. 35), S. 25 ff.

39 So der Vorsitzende Jens Seipenbusch, zitiert nach Markus Wehner, Piraten mit Potential, in: FAS vom 5. Juli 2009, S. 2.

40 Matthias Heitmann, Piratenpartei: ändern oder kentern?, in: NovoArgumente 2010, Nr. 105, S. 10 - 11, http://www.novo-argumente.com/magazin.php/archiv/novo105_10 (Abruf am 25. Oktober 2010). 
grundlegenden „Konflikt zwischen den neuen, gewachsenen Möglichkeiten durch die digitale Revolution und dem Regulierungsanspruch moderner Staatlichkeit“, wobei die Piraten dafür kämpfen, den Konflikt „im Sinne einer libertären Freiheitsauffassung aus Sicht der Nutzer zu lösen“" ${ }^{41}$.

Es spricht nichts dafür, die Frage nach der Ausgestaltung der Netzpolitik als eine neue zentrale gesellschaftliche Konfliktlinie anzusehen, da sie problemlos in das existierende sozio-kulturelle Cleveage zwischen libertären und autoritären Wertesystemen eingeordnet werden kann. ${ }^{42}$ Warum konnte dann aber eine neue Partei auf der libertären Seite dieser Konfliktlinie gegründet werden und die ersten beiden Karrierestufen überwinden, wenn diese Seite doch vor allem von den Grünen als Polpartei, aber auch von der FDP und der Linkspartei schon parteipolitisch repräsentiert ist? ${ }^{43}$ Die Antwort wird erleichtert, wenn man sich die Wähler der Piraten näher ansieht. Ihre bekannten sozialstrukturellen Merkmale (vgl. Tabelle 2) stimmen mit denen einer über ihre Medienmentalität - das heißt ihre Einstellungen zu Medien, ihren Interessen und Präferenzen und ihrem tatsächlichen Medienverhalten - definierten Personengruppe überein, die als „digital natives“ bezeichnet wird. ${ }^{44}$ Diese in der digitalen Welt aufgewachsene Gruppe bildet eine über das Internet vermittelte „deterritoriale Gemeinschaft “ 45 , deren aus den geteilten Netzinteressen gespeister gemeinsamer Sinnhorizont ein subjektiv gefühltes Zusammengehörigkeitsgefühl stiftet. Da die etablierten Parteien es versäumt haben, die digitale Revolution und ihre Probleme rechtzeitig aufzugreifen und zu diskutieren, fühlte sich jener Teil der digital natives, der die Netzpolitik als ausschlaggebenden Faktor der politischen Präferenzbildung ansah, in seiner Themenpräferenz und Interessenstruktur immer weniger politisch integriert und repräsentiert. Infolgedessen spricht man den etablierten Parteien die Netz-Kompetenz ab und sieht in der Piratenpartei die einzig legitime politische Vertretung. Daher werden es die etablierten Parteien trotz der jetzt erfolgten Integration netzpolitischer Themen in ihre Agenda sehr schwer haben, diese Wählergruppe zurückzugewinnen. Als reine Interessenvertretungspartei der netzpolitikaffinen digital natives werden die Piraten jedoch über die zweite Karrierestufe nicht hinauskommen, da die überwältigende Mehrheit der Wahlberechtigten entweder andere Themenpräferenzen hat oder gar nicht themenbezogen wählt. Daher wird in der Partei schon seit der Bundestagswahl eine sehr kontroverse Diskussion über die Art und das Ausmaß der thematisch breiteren Aufstellung geführt. Durch eine zu frühe inhaltlich-programmatische Verbreiterung gerät man jedoch in die Gefahr, den Markenkern der

41 Udo Zolleis / Simon Prokopf/ Fabian Strauch, a.a.O. (Fn. 35), S. 10 f.

42 Zur Konfliktstruktur vgl. Oskar Niedermayer, Gesellschaftliche und parteipolitische Konfliktlinien, in: Steffen Kühnel I ders. / Bettina Westle (Hrsg.), Wähler in Deutschland. Sozialer und politischer Wandel, Gender und Wahlverhalten, Wiesbaden 2009, S. 30 - 67.

43 Zur programmatischen Verortung der Piraten auf der sozio-kulturellen Konfliktlinie in der Nähe der drei genannten Parteien vgl. Marc Debus, Die Piratenpartei in der ideologischen Parteienkonstellation Deutschlands, http://blog. zeit.de/politik nach zahlen/2009/09/16/die-piratenpartei-inder-ideologischen-parteienkonstellation-deutschlands1961 (Abruf am 16. September 2009).

44 Vgl. Sinus Sociovision / Stern, Medienmentalitäten heute: Zwischen Virtualisierung und Bodenhaftung, in: Stern Anzeigenabteilung (Hrsg.), MarkenProfile 12. Neue Medienmentalitäten. Wohin geht die Zielgruppe?, Hamburg 2007.

$45 \mathrm{Zu}$ diesem Konzept vgl. Andreas Hepp, Medienkommunikation und deterritoriale Vergemeinschaftung. Medienwandel und die Posttraditionalisierung von translokalen Vergemeinschaftungen, in: Ronald Hitzler / Anne Honer / Michaela Pfadenhauer (Hrsg.), Posttraditionale Gemeinschaften, Wiesbaden 2008, S. 132 - 150. 
Partei zu verwischen, bevor er sich über die digital natives hinaus bei einer breiteren Bevölkerungsschicht überhaupt etabliert hat. Zudem bleibt abzuwarten, ob - über die LiquidFeedback-Diskussion - eine Positionierung nicht nur auf der sozio-kulturellen Libertarismus/Autoritarismus-Konfliktlinie, sondern auch auf der für die breite Mehrheit der Bevölkerung wichtigeren ökonomischen Sozialstaatskonfliktlinie zwischen sozialer Gerechtigkeit und Marktfreiheit gefunden werden kann, die sich hinreichend von den Konkurrenzparteien abgrenzt.

\subsection{Die Rahmenbedingungen des Parteienwettbewerbs}

Die Orientierungen und Handlungen der Akteure auf der Angebots- und Nachfrageseite des politischen Wettbewerbs werden durch eine Reihe von Rahmenbedingungen beeinflusst, die den Erfolg einer neuen Partei wie der Piraten entweder behindern oder fördern. Hierzu gehören der rechtliche Rahmen, die Situation im übrigen intermediären System und eine Reihe von sonstigen Bedingungen, die im Folgenden jedoch aus Platzgründen außer Acht gelassen werden.

Aus den Regelungen des Parteien- und Wahlrechts ergeben sich drei Hürden für den Erfolg neuer politischer Vereinigungen. Schon das Überschreiten der ersten Karrierestufe, also die Anerkennung als Partei, ist nicht einfach. Bei der Bundestagswahl 2009 haben 49 „sonstige politische Vereinigungen“, das heißt Bewerber, die im Bundestag oder einem Landtag seit deren letzter Wahl nicht ununterbrochen mit mindestens fünf Abgeordneten vertreten waren, eine Beteiligungsanzeige eingereicht. Nur 21 davon wurden im Juli 2009 vom Bundeswahlausschuss als Parteien anerkannt. ${ }^{46}$ Die Machtfülle des Bundeswahlausschusses bei der Zulassung neuer beziehungsweise kleiner Parteien stößt immer wieder auf verfassungsrechtliche Bedenken, da (1) er von einer der Kontrolle des Bundesinnenministers unterstellten Person geleitet wird (der Bundeswahlleiter wird vom Bundesinnenminister ernannt und kann auch jederzeit abberufen werden), (2) seine Zusammensetzung von den etablierten Parteien dominiert wird (die acht Beisitzer werden von den acht bei der letzten Bundestagswahl erfolgreichsten Parteien vorgeschlagen), und (3) die Unbestimmtheit des Maßstabes zur Zuerkennung der Parteieigenschaft ${ }^{47}$ dem Gremium einen sehr weiten Entscheidungsspielraum lässt. Daher urteilt zum Beispiel Meinel, dass es sich bei dem Zulassungsverfahren „um nicht anderes als ein Kooptationsverfahren“ handele: „Wer in Deutschland bei Wahlen als Partei antreten darf, entscheidet im Streitfall die unmittelbare Konkurrenz. "48 Hinzu kommt, dass die neue Partei in jedem Bundesland, in dem sie an der Wahl teilnehmen will, ihrer eingereichten Landesliste eine Liste mit Unterstützungsunterschriften von einem Tausendstel der Wahlberechtigten dieses Landes bei der letzten Bundestagswahl (höchstens jedoch 2.000) beilegen muss, was den Piraten durch ihre Werbeaktionen im Internet leichter

46 Vgl. Bundeswahlleiter, Pressemitteilungen Nr. 38 vom 30. Juni und Nr. 41 vom 17. Juli 2009.

47 Es muss beurteilt werden, ob die Vereinigung nach dem „Gesamtbild der tatsächlichen Verhältnisse“ (\$ 2 I PartG) eine ausreichende Gewähr für die Ernsthaftigkeit ihrer Ziele bietet, auf die politische Willensbildung Einfluss zu nehmen und parlamentarisch an der Vertretung des Volkes mitzuwirken.

48 Florian Meinel, Chancengleichheit oder Kooptation? Der Zugang kleiner Parteien zur Bundestagswahl, in: ZParl, 41. Jg. (2010), H. 1, S. 67 - 76, S. 71. 
fällt als anderen Kleinstparteien, die ihre wenigen Mitglieder auf die Straße zum Unterschriften sammeln schicken müssen. Nicht vergessen werden darf auch, dass die genannten Hürden - so lange die Partei nicht parlamentarisch repräsentiert ist - bei jeder Wahlteilnahme erneut genommen werden müssen. Eine politische Vereinigung kann daher bei einer Bundestagswahl als Partei anerkannt werden, bei der nächsten jedoch nicht.

Das zweite erfolgsrelevante Hindernis ist die Erfüllung der Anspruchsberechtigung für die öffentliche Teilfinanzierung. Anspruchsberechtigt in einem bestimmten Jahr sind alle Parteien, die bei der letzten Bundestags- oder EP-Wahl mindestens 0,5 Prozent oder bei einer der letzten 16 Landtagswahlen mindestens 1,0 Prozent der abgegebenen gültigen Zweitstimmen erhalten haben. Die Überwindung dieser Hürde bedeutet zwar nicht das Überschreiten einer Karrierestufe, schafft aber günstige Voraussetzungen dafür, weil sie der Partei dringend benötigte finanzielle Ressourcen zuführt, wobei deren Höhe durch die eigenen Einnahmen begrenzt wird (vgl. Abschnitt 3.1.). Das dritte, öffentlich am deutlichsten sichtbare Hindernis besteht in der Regelung des Bundeswahlgesetzes, wonach nur Parteien an der Mandatsverteilung für den Bundestag teilnehmen, die mindestens 5 Prozent der abgegebenen gültigen Zweitstimmen erhalten haben. Eingeführt, um einer zu starken Zersplitterung des Parlaments und den damit verbundenen möglichen Problemen bei der Regierungsbildung und -stabilität vorzubeugen, bildet die Fünfprozentklausel eine relativ hohe Hürde für das Überschreiten der dritten Karrierestufe, also die parlamentarische Repräsentation.

Eine weitere rechtliche Rahmenbedingung des Parteienwettbewerbs ist die föderale Staatsorganisation der Bundesrepublik, die es neuen kleinen Parteien mit einem klaren regionalen Politikbezug oder regionalen Organisations- und Wählerschwerpunkten erleichtert, die parlamentarische Repräsentationsschwelle in einem Bundesland zu überwinden beziehungsweise durch deren Überwindung die Chancen für ein bundesweites Überschreiten dieser Karrierestufe zu verbessern. Auch die Piraten haben durchaus regionale Organisations- und Wählerschwerpunkte. Sie weisen zum Beispiel in den beiden großstädtischen Bundesländern Hamburg und Berlin die größte Organisationsdichte auf und haben dort auch bei der EP- und Bundestagswahl die besten Ergebnisse erzielt, so dass die Partei zum Beispiel unter sonst gleichen Umständen bei der Abgeordnetenhauswahl in Berlin im Herbst 2011 besser abschneiden könnte, als es bei der Landtagswahl in Nordrhein-Westfalen 2010 der Fall war. Auch in Berlin ist jedoch ein Überschreiten der Fünfprozenthürde aus heutiger Sicht sehr unwahrscheinlich.

Neben den rechtlichen Regeln ist die Situation im intermediären System für eine neue Partei relevant, weil ihre Erfolgschancen durch das Ausmaß an Unterstützung beeinflusst werden, die sie durch Medien, Verbände und soziale Bewegungen erfährt, wobei vor allem „das Überschreiten der Selektionsschwelle der Medien ... eines der Hauptprobleme “ 49 darstellt. Für die Piraten lassen sich in diesem Bereich zumindest in neuester Zeit keine wirklich erfolgsfördernden Rahmenbedingungen mehr erkennen. Die Partei kann „weder auf die Unterstützung existierender Großorganisationen noch auf eine Art von einer Neuen Sozialen Bewegung, die die Freiheit des Internets verteidigen wollte, zurückgreifen " 50 . Die Medienunterstützung verlief sehr unterschiedlich, wie Abbildung 2 am Beispiel der Presseberichterstattung zeigt.

49 Marcel Solar, a.a.O. (Fn. 39), S. 109.

50 Udo Zolleis / Simon Prokopf/ Fabian Strauch, a.a.O. (Fn. 35), S. 17. 


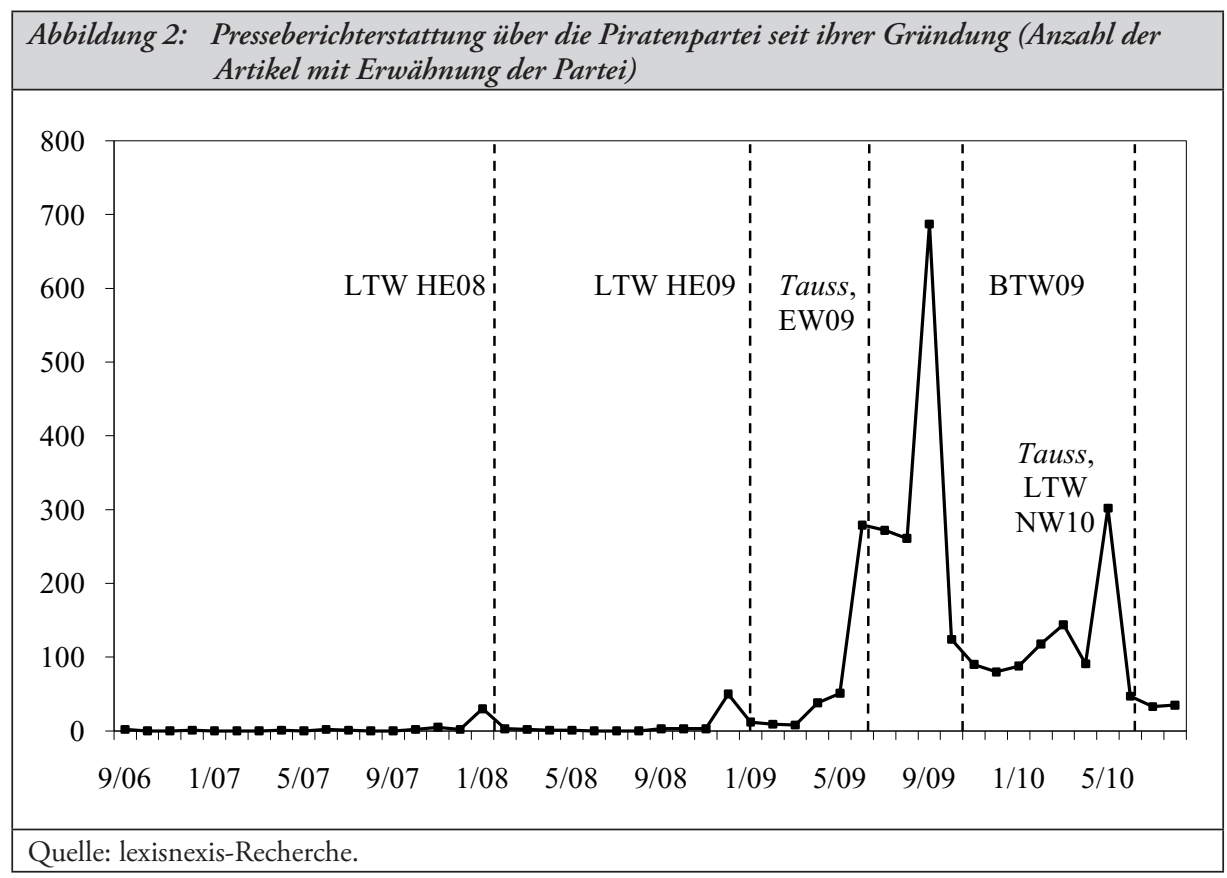

Nach ihrer Gründung wurde die Partei lange Zeit nicht wahrgenommen, lediglich ihre beiden Landtagswahlteilnahmen erreichten ein wenig Medienaufmerksamkeit. Dies änderte sich erst ab dem Juni 2009 mit den Berichterstattungen über die Affäre Tauss, den Achtungserfolg bei der EP-Wahl, das Netzsperrengesetz und den Bundestagswahlkampf. Nach der Bundestagswahl wurde es deutlich ruhiger um die Piraten, und ein erneutes Hoch schaffte erst wieder die Verurteilung von Tauss und die Landtagswahl in NRW. Danach war die Partei wieder fast kein Thema mehr. Zudem hat sich der Tenor der Berichterstattung gewandelt. Profitierten die Piraten 2009 von ihrem Neuigkeitswert und wurden generell positiv dargestellt, so taugten sie in neuerer Zeit nicht mehr als Feuilletonaufmacher. Ihre internen Probleme wurden stärker thematisiert, und nach ihrem relativ schlechten Abschneiden in Nordrhein-Westfalen wurde ihnen in manchen Beiträgen schon das Totenglöcklein geläutet. ${ }^{51}$

Unter den Rahmenbedingungen sowie auf der Angebots- und Nachfrageseite des politischen Wettbewerbs gibt es also eine ganze Reihe von Hemmfaktoren, die den Erfolg neuer Parteien im bundesrepublikanischen Parteiensystem behindern. Auch die Piratenpartei ist bisher über die zweite Karrierestufe nicht hinaus gekommen, und es spricht vor allem auf der Bundesebene wenig dafür, dass dies in absehbarer Zeit gelingt.

51 „Es war einmal die Piratenpartei.“ Ole Reißmann, Piraten erleiden Schiffbruch, in: Spiegel online vom 24. Mai 2010, http://www.spiegel.de/politik/deutschland/0,1518,695167,00.html (Abruf am 25. Oktober 2010). 